This item was submitted to Loughborough's Research Repository by the author.

Items in Figshare are protected by copyright, with all rights reserved, unless otherwise indicated.

\title{
Synaptically generated wave propagation in excitable neural media
}

PLEASE CITE THE PUBLISHED VERSION

PUBLISHER

(C) American Physical Society

LICENCE

CC BY-NC-ND 4.0

REPOSITORY RECORD

Bressloff, P.C.. 2019. "Synaptically Generated Wave Propagation in Excitable Neural Media". figshare. https://hdl.handle.net/2134/1736. 


\title{
Synaptically Generated Wave Propagation in Excitable Neural Media
}

\author{
P. C. Bressloff \\ Nonlinear and Complex Systems Group, Department of Mathematical Sciences, Loughborough University, \\ Loughborough, Leicestershire, LE11 3TU, United Kingdom
}

(Received 26 October 1998)

\begin{abstract}
We study the propagation of solitary waves in a one-dimensional network of excitable integrate-andfire neurons with axo-dendritic synaptic coupling. We show that for small axonal delays there exists a stable solitary wave, and derive a power scaling law for the velocity as a function of the coupling. In the case of large axonal delays and fast synapses we establish that the solitary wave can destabilize via a Hopf bifurcation in the firing times. [S0031-9007(99)08852-3]
\end{abstract}

PACS numbers: 87.10.+e, 05.45.-a

Many cells in nature are excitable in the sense that a sufficiently strong stimulus will induce the membrane potential of the cell to undergo a large excursion, known as an action potential, before coming back to rest. Examples include cardiac cells, smooth muscle cells, secretory cells, and most neurons. Excitability is a useful property since it confers on a cell the ability to transmit signals reliably in the presence of noise. An interesting feature of spatially extended excitable systems is that they can support the propagation of solitary waves in the form of traveling fronts and pulses and, in the case of two-dimensional networks, more complex geometrical structures such as spirals. (For a recent review, see Ref. [1] and references therein.) Most studies of excitable waves assume that the underlying mechanism for wave propagation is diffusive in nature, as exemplified by an action potential traveling along an axon. However, a number of recent experimental studies have revealed the propagation of synaptically generated waves in slices of excitable neural tissue taken from the cortex [2,3], hippocampus [4,5], and thalamus [6]. Such waves have also been observed in detailed computational models of neural tissue treated as a one-dimensional continuum [3-5,7]. A simplified integrate-and-fire (IF) version of these models has been analyzed by Ermentrout [8], who derived a self-consistency condition for the velocity of a solitary pulse and showed that there exists a fast solution branch and a slow solution branch. Moreover, the velocity of a fast wave scales as a power of the coupling strength. Although the stability of these waves was not addressed, numerical evidence suggested that the fast (slow) wave is stable (unstable).

In this Letter, we analyze the stability of solitary waves in a spatially distributed network of synaptically coupled excitable IF neurons. We prove that in the case of small axonal delays fast solitary waves are stable, whereas slow waves are unstable. Moreover, we establish that in the case of large axonal delays and fast synapses, a fast wave can itself destabilize due to a Hopf bifurcation in the firing times. To formulate the basic problem, consider a one-dimensional network of IF neurons with excitatory coupling evolving according to the equation

$$
\frac{\partial V(x, t)}{\partial t}=I_{0}-\frac{V(x, t)}{\tau_{m}}+I(x, t) .
$$

Here $V(x, t)$ denotes the membrane potential of the neuron at $x \in \mathbb{R}$ at time $t, \tau_{m}$ is the membrane time constant, $I_{0}$ is a constant external input, and $I(x, t)$ is the total synaptic input into the cell. Equation (1) is supplemented by the reset condition $V\left(x, t^{+}\right)=0$ whenever $V(x, t)=h$, where $h$ is the threshold for firing. We set $h=1$ and $\tau_{m}=1$ for convenience. This fixes the units of time to be of the order of 1-10 msec. In the absence of any synaptic inputs, we can distinguish between an oscillatory regime $\left(I_{0}>1\right)$ in which each neuron independently fires at regular intervals of period $T_{0}=\ln \left(I_{0} /\left[I_{0}-1\right]\right)$, and an excitable regime $\left(I_{0}<1\right)$ in which each neuron requires an additional stimulus before it can fire. We restrict ourselves here to the excitable regime by setting $I_{0}=0$. (Oscillatory IF networks are studied in some detail elsewhere [9].) The synaptic current is taken to be of the form

$$
I(x, t)=g \int_{-\infty}^{\infty} W\left(x-x^{\prime}\right) E\left(x^{\prime}, t\right) d x^{\prime},
$$

where $E(x, t)$ is the input at time $t$ due to the firing of the presynaptic neuron at $x, g$ is a positive coupling parameter, and $W(x)$ is taken to be positive, symmetric, and a monotonically decreasing function of $|x|$ with $\int_{0}^{\infty} W(x) \times$ $d x<\infty$. Typical choices for such a weight function are $W(x)=\left(2 \pi \sigma^{2}\right)^{-1 / 2} e^{-x^{2} / 2 \sigma^{2}}$ (Gaussian) and $W(x)=$ $(2 \sigma)^{-1} e^{-|x| / \sigma}$ (exponential). For concreteness, we choose the exponential function, although our basic results will not depend on the precise form of $W(x)$

We define a solitary wave solution of Eqs. (1) and (2) to be one for which

$$
E(x, t)=J(t-x / c),
$$

where $c$ is the velocity of the wave and $J(t)$ is the wave profile with $J(t)=0$ for all $t \leq 0$. The physical interpretation of such a solution is that each neuron fires once with the neuron at $x$ firing at time $T(x)=x / c$ (up to an arbitrary constant reflecting translation invariance). The 
function $J(t)$ represents the linear response of a neuron to an incoming spike idealized as a Dirac delta-function (see below). In order to determine the velocity $c$ of a solitary pulse, we substitute Eqs. (2) and (3) into Eq. (1), and then integrate with respect to $t$ over the interval $-\infty<t \leq$ $T(x)=x / c$. This generates a self-consistency condition for $c$ of the form

$$
1=g \int_{0}^{\infty} W(x) e^{-x / c} \int_{0}^{x / c} e^{t} J(t) d t d x .
$$

This is equivalent to the self-consistency condition derived in Ref. [8]. We can explicitly integrate over $x$ and $t$ in Eq. (4) by Fourier expanding $J(t)$ and using the analyticity properties of $\tilde{J}(\omega)=\int_{-\infty}^{\infty} e^{-i \omega t} J(t) d t$, namely, that $\widetilde{J}(\omega)$ is analytic in the lower-half complex $\omega$ plane since $J(t)=$ 0 for $t \leq 0$. After performing a contour integral we arrive at the useful result

$$
1=\frac{g c}{2(1+c)} \tilde{J}(-i c) .
$$

The (local) asymptotic stability of a solitary wave can be determined by considering perturbations of the firing times [10]. Suppose that $T(x)=x / c+u(x)$ with $u(x)=\phi(x)$ for $x \leq 0$ and $\phi(x)$ a prescribed, bounded function on $(-\infty, 0]$. Asymptotic stability then corresponds to the condition $u(x) \rightarrow 0$ as $x \rightarrow \infty$ for arbitrary nonuniform initial data $\phi(x)$. Integrate Eq. (1) over the interval $(-\infty, T(x)]$ and expand the resulting nonlinear integral equation to first order in $u(x)$. Substitution of a solution of the form $u(x)=e^{\lambda x}$ into the linearized integral equation then yields the characteristic equation

$$
\int_{0}^{\infty}\left[e^{-\lambda x}-1\right] W(x) f(x / c) d x=0,
$$

where

$$
f(\tau)=\int_{0}^{\tau} e^{-(\tau-t)} J^{\prime}(t) d t,
$$

and $J^{\prime}$ denotes differentiation with respect to $t$. Asymptotic stability holds if all nonzero solutions $\lambda$ of Eq. (6) have a negative real part. Such solutions depend indirectly on the coupling $g$ via the speed $c$, which satisfies Eq. (5). Equation (6) can be evaluated along identical lines to the analysis of Eq. (5). If we set $\lambda=a+i b$ and expand equation (6) into real and imaginary parts, then we obtain the pair of equations

$$
\begin{gathered}
H_{1}(a, b) \equiv \operatorname{Re} P(c+a+i b)-P(c)=0, \\
H_{2}(a, b) \equiv-\operatorname{Im} P(c+a+i b)=0,
\end{gathered}
$$

where

$$
P(z)=\frac{z}{(1+z)} \tilde{J}(-i z) .
$$

Note that $P(z)$ is real when $z$ is real, and $P(z) \rightarrow 0$ as $|z| \rightarrow \infty$.
It is clear from the above analysis that both the existence and stability of a solitary pulse depend crucially on the properties of the linear response function $J(t)$. There are a number of possible contributions to $J(t)$. These include the finite transmission time for the propagation of a spike along an axon, the time-dependent opening and closing of ionic conductance channels in the postsynaptic membrane, and diffusion of the postsynaptic potential along the dendritic tree of a neuron. These various features can be taken into account at the simplest level by assuming that $J(t)$ has the general form

$$
J(t)=J_{0}\left(t-\tau_{a}\right) \theta\left(t-\tau_{a}\right),
$$

where $\tau_{a}$ is a typical discrete axonal delay, $\Theta(t)=1$ if $t \geq 0$ and is zero otherwise, and $J_{0}(t), t \geq 0$, is a unimodal function of time with $J_{0}(0)=0$. We denote the time at which $J_{0}(t)$ reaches its maximum by $\tau_{\max }, \tau_{\max }>$ 0 , and refer to the synaptic coupling as either fast or slow according to whether $\tau_{\max }$ is small or large. A common representation of the time-dependent variation in membrane conductance is the so-called alpha function, for which $J_{0}(t)=\alpha^{2} t e^{-\alpha t}$ where $\alpha$ is the inverse rise time with the time to peak $\tau_{\max }=\alpha^{-1}$. (This particular case was studied in Ref. [8].) The effects of dendritic processes can be incorporated by taking $J_{0}(t)$ to be the Green's function of the associated cable equation. The latter describes the diffusive effects of passive membranes on the spread of synaptic currents along the dendritic tree of a neuron [11]. Suppose, for simplicity, that each neuron consists of a soma or cell body where spike generation occurs, which is connected resistively to the end of a semi-infinite uniform dendritic cable. As a further simplification, suppose that all synapses are located at a fixed position $\xi_{0}, 0 \leq \xi_{0}<\infty$ on the dendritic cable independently of the positions of the interacting neurons. Solving the resulting cable equation then leads to the result

$$
J_{0}(t)=G\left(\xi_{0}, t\right) \equiv \frac{e^{-t / \tau_{d}}}{\sqrt{\pi D t}} e^{-\xi_{0}^{2} / 4 D t},
$$

where $\tau_{d}$ is the membrane time constant of the cable and $D$ is the diffusivity. The Green's function $G(\xi, t)$ determines the membrane potential response at the end of the cable due to an instantaneous injection of unit current at point $\xi$ at time $t$. It follows from Eq. (12) that $\tau_{\max }=\tau_{d}\left[-1+\sqrt{1+4 \xi_{0}^{2} /\left(D \tau_{d}\right)}\right] / 4$.

We now investigate the existence and stability of solitary pulses as a function of the time to peak $\tau_{\max }$ of the dendritic response function (12) and the axonal delay $\tau_{a}$. First, Fourier transform Eqs. (11) and (12) to obtain

$$
\tilde{J}(\omega)=\frac{1}{\sqrt{1+i \omega}} e^{-\sqrt{1+i \omega} \xi_{0}} e^{-i \omega \tau_{a}},
$$

where we have set $\tau_{d}=D=1$. Using Eqs. (5) and (13), we plot solutions $c(g)$ for various values of $\xi_{0}$ and $\tau_{a}$ in Fig. 1. It can be seen that there exists a critical 


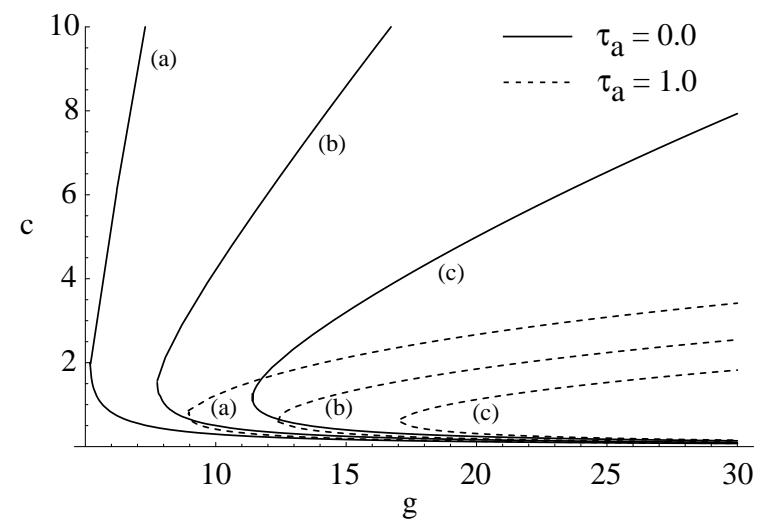

FIG. 1. Variation of the speed $c$ of a traveling pulse (in units of $\sqrt{D / \tau_{d}}$ ) as a function of the synaptic coupling $g$ for different values of the dendritic coordinate $\xi_{0}$ (in units of $\sqrt{D \tau_{d}}$ ) and axonal delay $\tau_{a}$. (a) $\xi_{0}=0.0$; (b) $\xi_{0}=0.1$; (c) $\xi_{0}=0.5$. For fixed $\tau_{a}$ and $\xi_{0}$, there exists a fast solution branch $C_{+}$and a slow solution branch $C_{-}$.

coupling $g=g_{s}\left(\xi_{0}, \tau_{a}\right)$ such that there are no solitary wave solutions for $g<g_{s}$ and two solutions for $g>g_{s}$. Moreover, when $\xi_{0}=0$ (synapses are proximal to the soma) and $\tau_{a}=0$, the velocity exhibits a power scaling law given by $c \sim g^{2}$ for large $c$. This contrasts with the square-root law $c \sim \sqrt{g}$ found previously for synaptic delays [8].

The stability of the fast and slow solution branches displayed in Fig. 1 can be determined from the following theorem:

Theorem 1.- - Let $C_{+}$and $C_{-}$denote, respectively, the fast and slow solution branches $c=c_{ \pm}(g), g \geq g_{s}$, of the self-consistency condition (5) for the velocity of a solitary pulse, assuming a unimodal response function $J(t)$ satisfying Eq. (11). Here $g_{s}$ is the critical coupling for the existence of a solitary wave. Denote the time to peak of $J_{0}(t)$ by $\tau_{\max }$. The following stability results then hold: (i) The branch $C_{-}$is unstable for all $\tau_{\max }$ and $\tau_{a}$. (ii) The branch $C_{+}$is stable for all $\tau_{\max }$ in the case of zero axonal delays, $\tau_{a}=0$. (iii) In the case of large axonal delays and fast synapses (small $\tau_{\max }$ ), there exists a Hopf bifurcation point $g_{h}$ such that $c_{+}(g)$ is stable (unstable) for $g>g_{h}$ $\left(g_{s} \leq g<g_{h}\right)$.

We shall sketch the proof of this theorem. First, differentiation of Eq. (8) with respect to $a$ and Eq. (5) with respect to $c$ shows that $g^{2} \partial H_{1}(a, 0) /\left.\partial a\right|_{a=0}=-c^{2} d g / d c$. Hence, $\partial H(a, 0) /\left.\partial a\right|_{a=0}>0$ when $d g / d c<0$, that is, when $c \in C_{-}$. Since $\lim _{a \rightarrow \infty} H_{1}(a, 0) \equiv-P(c)<0$, it follows that $H(a, 0)$ must cross the positive $a$ axis at least once when $c \in C_{-}$. This implies that $H(a, 0)=0$ has at least one solution for $a>0$ and, hence, that the branch $C_{-}$is unstable. Second, since the delay kernel $J_{0}(t)$ of Eq. (11) is unimodal with a maximum at $\tau_{\max }$, it follows that the function $f(\tau)$ of Eq. (6) is also unimodal with a maximum at $\tau=\tau_{\max }$ such that $f(\tau)>0$ for $0<\tau<$ $\tau_{\max }$. Since $W(x)$ is a monotonically decreasing func- tion of $x$, it follows from Eq. (8) that $H_{1}(a, b)<0$ for all $a>0$ and $b \geq 0$ when $c \tau_{\max }$ is sufficiently large. In other words, the branch $C_{+}$is stable in the large- $c$ limit. It can also be established that $H(a, 0)=0$ does not have any positive definite solutions when $c \in C_{+}$so that the upper branch is stable with respect to static bifurcations. The proof is completed by establishing conditions for the occurrence of a Hopf instability on the upper branch. This requires searching for solutions $a=0, b \neq 0$ of Eqs. (8) and (9) [12]. We shall carry this out explicitly for $J_{0}(t)$ given by Eq. (12) and $\tau_{\max } \rightarrow \xi_{0}$. Similar results hold for an alpha-function response with $\tau_{\max } \rightarrow \alpha^{-1}$.

Let $c_{h}$ denote the largest velocity for which a solution $a=0, b \neq 0$ of Eqs. (8) and (9) exists. Also set $c_{s}=$ $c_{ \pm}\left(g_{s}\right)$, which is the critical velocity at which the two solution branches $C_{ \pm}$meet. In Fig. 2, we plot the critical velocities $c_{s}\left(\xi_{0}\right)$ and $c_{h}\left(\xi_{0}\right)$ as a function of the dendritic coordinate $\xi_{0}$ and various delays $\tau_{a}$. For a given delay $\tau_{a}$, $c \in C_{+}$if $c>c_{s}\left(\xi_{0}\right)$ and $c \in C_{-}$if $c<c_{s}\left(\xi_{0}\right)$. It can be seen from Fig. 2 that for $\tau_{a}>\bar{\tau}_{a} \approx 0.5$ there exists a critical point $\bar{\xi}_{0}$ such that $c_{s}\left(\xi_{0}\right)>c_{h}\left(\xi_{0}\right)$ for $\xi_{0}>\bar{\xi}_{0}$ and $c_{s}\left(\xi_{0}\right)<c_{h}\left(\xi_{0}\right)$ for $\xi_{0}<\bar{\xi}_{0}$. The crossover points in the particular cases $\tau_{a}=0.75$ and $\tau_{a}=1.0$ are labeled by $A$ and $B$, respectively. Suppose that we now reduce $c$ or, equivalently $g$, along the branch $C_{+}$for fixed $\xi_{0}$ and $\tau_{a}>0$. For $\xi_{0}<\bar{\xi}_{0}$ (fast synapses), we find that a Hopf bifurcation occurs at a critical coupling $g_{h}$ such that $c_{+}\left(g_{h}\right)=c_{h}\left(\xi_{0}\right)$ and solitary waves are unstable for all $c<c_{h}\left(\xi_{0}\right)$. On the other hand, if $\xi_{0}>\bar{\xi}_{0}$ (slow synapses) then the branch $C_{+}$remains stable until it merges with the unstable lower branch $C_{-}$at $c=c_{s}\left(\xi_{0}\right)$.

Our analysis of solitary pulses is easily extended to two-dimensional networks. Consider a plane wave propagating in the direction $\mathbf{n}$ with speed $c$. That is, the neuron

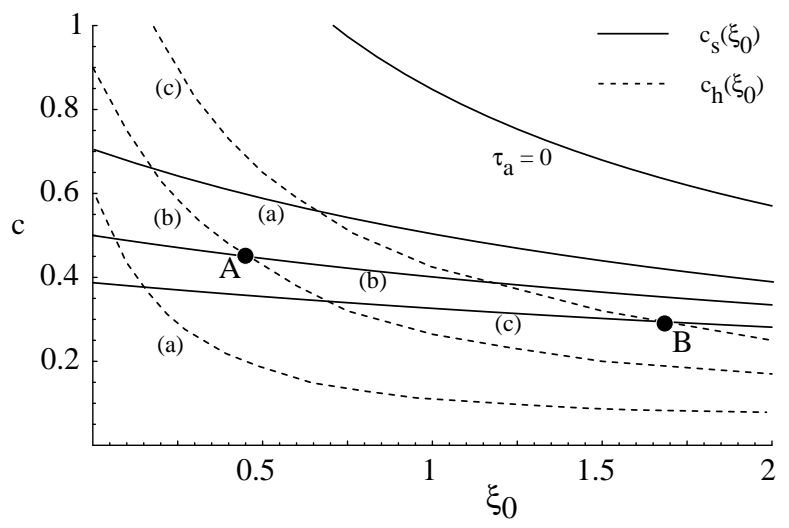

FIG. 2. Plot of critical velocities $c_{s}\left(\xi_{0}\right)$ (solid curves) and $c_{h}\left(\xi_{0}\right)$ (dashed curves) as a function of the dendritic coordinate $\xi_{0}$ and various axonal delays $\tau_{a}$. (a) $\tau_{a}=0.5$; (b) $\tau_{a}=0.75$; (c) $\tau_{a}=1.0$. The critical velocity $c_{s}\left(\xi_{0}\right)$ determines the point at which the fast and slow wave branches $C_{ \pm}$of Fig. 1 bifurcate. For fixed $\tau_{a}$ and $\xi_{0}$, the fast branch $C_{+}$undergoes a Hopf bifurcation at the critical velocity $c_{h}\left(\xi_{0}\right)$ if and only if $c_{h}\left(\xi_{0}\right)>c_{s}\left(\xi_{0}\right)$. Same units as Fig. 1 . 
at position $\mathbf{r}=(x, y)$ fires once at time $T(\mathbf{r})=\mathbf{n} \cdot \mathbf{r} / c$. The speed $c$ satisfies the self-consistency condition [see Eq. (4)]

$$
1=g \int_{\mathbb{R}} W(|\mathbf{r}|) e^{-\mathbf{n} \cdot \mathbf{r} / c} \Theta(\mathbf{n} \cdot \mathbf{r}) \int_{0}^{\mathbf{n} \cdot \mathbf{r} / c} e^{t} J(t) d t d^{2} \mathbf{r} .
$$

The stability of such a wave can be analyzed by considering perturbations of the firing times as in the derivation of Eq. (6). Suppose that $\mathbf{n}=(1,0)$ and take $T(\mathbf{r})=$ $x / c+u(\mathbf{r})$. Linearizing the resulting integral equation for the perturbations $u(\mathbf{r})$ and taking $u(\mathbf{r})=e^{\lambda x+i \mathbf{p} \cdot \mathbf{r}}$, $\mathbf{p}=\left(p_{1}, p_{2}\right)$, we obtain a characteristic equation for $\lambda$ :

$$
\int_{\mathbb{R}} W(|\mathbf{r}|)\left(e^{-\lambda x-i \mathbf{p} \cdot \mathbf{r}}-1\right) f(x / c) \Theta(x) d^{2} \mathbf{r} .
$$

For reasonable choices of the weight kernel $W$ one finds fast and slow wave solutions of Eq. (14) along similar lines to the one-dimensional case, and the stability results of theorem 1 still hold.

Another interesting extension of our work is to the case of periodic wave trains. In one dimension, these correspond to solutions of Eqs. (1) and (2) of the form $E(x, t)=\sum_{m \in \mathbb{Z}} J[t-(m+k x) T]$ where $T$ is the period of the wave and $k$ is the wave number. The speed of the wave is $c=(k T)^{-1}$. Such a solution can also be interpreted as a phase-locked state in which all the neurons fire at regular intervals $T$ such that the $m$ th firing time of the neuron at $x$ is $T_{m}(x)=(m+k x) T$ [9]. Solving Eqs. (1) and (2) generates dispersion curves of the form $T=T(k)$. It turns out that if $g>g_{s}$ where $g_{s}$ is the critical coupling for the existence of solitary pulses (see, e.g., Fig. 1), then there are three corresponding dispersion curves. Two of these are the continuations of the fast and slow solitary wave solutions, which are recovered in the long-wavelength limit $k \rightarrow 0, T \rightarrow \infty$ such that $\lim _{k \rightarrow c} c(k)=c$, where $c$ is the velocity of a solitary wave satisfying Eq. (5). The third dispersion curve is the continuation of the synchronous state $k=0$. This will be discussed in more detail elsewhere [13].

We end by briefly discussing some related work on waves in excitable two-dimensional neural systems. Kistler et al. have recently analyzed solitary waves and periodic wave trains in an excitable network based on the so-called spike response model [14], which is essentially an integrated version of the IF model studied in this Letter. A number of differences emerge between their treatment and our own. First, they effectively take the activity of a solitary pulse propagating in the $x$ direction to be $E(x, t)=c^{-1} J(t-x / c)$ rather than Eq. (3). The additional factor of $c^{-1}$ results in only one solution branch for $c=c(g)$ rather than two as found by ourselves and by Ermentrout [8]. It is not clear that there is any biophysical justification for this additional factor given the interpretation of $J(t)$ as an impulse response function. Second, their stability analysis is mainly based on studying changes in local field potentials, which is not sufficient to guarantee asymptotic stability with respect to more general perturbations of the firing times as considered here. In particular, it does not take into account the full spectrum of the linearized firing time map, which is crucial in establishing the occurrence of Hopf instabilities (see also Ref. [9]). The numerical results presented in Ref. [14] do, however, reveal a rich repertoire of geometrical waves such as spirals and target patterns, which certainly warrant further study. (Spiral waves have also been generated in IF networks [15].)

[1] J.P. Keener and J. Sneyd, Mathematical Physiology (Springer-Verlag, New York, 1998).

[2] R. D. Chervin, P. A. Pierce, and B. W. Connors, J. Neurophysiol. 60, 1695-1713 (1988).

[3] D. Golomb and Y. Amitai, J. Neurophysiol. 78, 11991211 (1997).

[4] R. D. Traub, J. G. R. Jeffreys, and R. Miles, J. Physiol. (London) 472, 267-287 (1993).

[5] D. Golomb, X. J. Wang, and J. Rinzel, J. Neurophysiol. 75, 750-769 (1996).

[6] U. Kim, T. Bal, and D. A. McCormick, J. Neurophysiol. 74, 1301-1323 (1995).

[7] A. Destexhe, T. Bal, D. A. McCormick, and T. J. Sejnowski, J. Neurophysiol. 76, 2049-2070 (1996).

[8] G. B. Ermentrout, J. Comp. Neurosci. 5, 191-208 (1998).

[9] P.C. Bressloff and S. Coombes, Phys. Rev. Lett. 80, 4815-4818 (1998); 81, 2168-2171 (1998); 81, 23842387 (1998).

[10] We restrict ourselves in this Letter to linear stability analysis based upon perturbations traveling at the same speed as the wave itself, that is, perturbations of the single neuron firing times $T(x)$. It is possible that there are further instabilities associated with global bifurcations where, for example, certain neurons fire more than once, fail to fire, or fire out of sequence.

[11] W. Rall, in Methods of Neuronal Modeling: From Synapses to Networks, edited by C. Koch and I. Segev (MIT Press, Cambridge, 1989), pp. 9-62.

[12] Under perturbations $\delta T(x) \sim e^{\lambda x}$ of the firing times we have $\delta E(x, t) \sim J^{\prime}(t-x / c) e^{\lambda x}$. Hence, we identify $\lambda= \pm i b$ as a Hopf bifurcation point since it results in a time-periodic modulation of the activity $E(x, t)$ in the coordinate frame of the solitary wave, that is, $\delta E(x, t) \sim A(x-c t) e^{i b c t}$. Linear stability analysis is itself insufficient to determine (i) whether the bifurcation is subcritical or supercritical and (ii) the new state generated by the instability.

[13] P. C. Bressloff (to be published).

[14] W. M. Kistler, R. Seitz, and J. L. van Hemmen, Physica (Amsterdam) 114D, 273-295 (1998).

[15] P. H. Chu, J. G. Milton, and J. D. Cowan, Int. J. Bifurcation Chaos. Appl. Sci. Eng. 4, 237 (1994). 